\title{
El reemplazo de los ácidos grasos saturados por ácidos grasos mono y poliinsaturados aporta beneficios en la salud
}

The replacement of saturated fatty acids by mono and polyunsaturated fatty acids brings health benefits

\section{Objetivo}

Establecer la relación entre el tipo de grasa ingerida y la mortalidad total y/o la mortalidad específica.

\section{Diseño}

Análisis de las bases de datos de dos estudios de cohorte: el Estudio de Salud de las Enfermeras (sigla en inglés NHS) y el Estudio de Seguimiento de Profesionales de la Salud (sigla en inglés HPFS).

\section{Lugar}

Once de los estados más poblados de Estados Unidos (California, Connecticut, Florida, Maryland, Massachusetts, Michigan, New Jersey, New York, Ohio, Pennsylvania y Texas).

\section{Pacientes}

Las cohortes incluyeron a 83.349 enfermeras de 30 a 55 años (seguidas entre 1980 a 2012) y 42.884 hombres profesionales de la salud de 40 a 75 años (seguidos entre 1986 y 2012), libres de enfermedad cardiovascular, cáncer y diabetes al inicio del seguimiento.

\section{Factores de riesgo evaluados}

Edad, sexo, nivel de actividad física, enfermedades metabólicas, patología cardíaca. La ingesta de grasas fue evaluada al inicio de la cohorte y fue actualizada cada dos a cuatro años.

\section{Medición de resultados principales}

Mortalidad total y mortalidad específica, obtenida de revisiones sistemáticas de registros vitales gubernamentales.

\section{Resultados principales}

Se observaron reducciones estadísticamente significativas en la mortalidad total al reemplazar el $5 \%$ de la energía proveniente de ácidos grasos saturados por ácidos grasos poliinsaturados (HR 0,73; IC95\% 0,70 a 0,77) y monoinsaturados (HR 0,87; IC95\% 0,82 a 0,93). Al comparar las personas en el quintilo de mayor consumo de grasas totales contra
Dong D y col. JAMA Intern Med. 2016;176(8):1134-1145. las del quintilo màs bajo, se observó además, una reducción significativa en la mortalidad total (HR 0,84; IC95\% 0,81 a 0,88), pero un aumento de la mortalidad cardiovascular (HR 1,20; IC96\% 1,08 a 1,33). Se analizó también el riesgo la mortalidad total de acuerdo al tipo de ácido graso consumido (ver tabla 1). Según los resultados observados, el alto consumo de ácidos grasos saturados y grasas trans aumentarían la mortalidad total; en cambio la ingesta de ácidos grasos mono y poliinsaturados, especialmente omega 6 , tendría una relación inversamente proporcional con la mortalidad total, lo que no se observó respecto de los ácidos grasos omega 3.

Tabla 1: Mortalidad de acuerdo al tipo de ácido graso consumido, al comparar las personas en el quintilo de mayor consumo con las del quintilo de menor consumo.

\begin{tabular}{c|c|c}
\hline Tipo de ácido graso & HR ajustado* $\mathbf{( I C 9 5 \% )}$ & $\mathbf{p}$ \\
\hline Saturado & $1,08(1,03 \mathrm{a} 1,14)$ & $<0,001$ \\
\hline Poliinsaturado & $0,81(0,78 \mathrm{a} 0,84)$ & $<0,001$ \\
\hline Monoinsaturado & $0,89(0,84 \mathrm{a} 0,94)$ & $<0,001$ \\
\hline Grasas Trans & $1,13(1,07 \mathrm{a} 1,18)$ & $<0,001$ \\
\hline Omega 6 & $0,85(0,81 \mathrm{a} 0,89)$ & $<0,001$ \\
\hline
\end{tabular}

*Ajustado por edad, estado matrimonial, raza, índice de masa corporal, actividad física, tabaquismo, consumo de alcohol, uso de suplementos vitamínicos, y antecedentes cardiovasculares.

\section{Conclusiones}

En concordancia con las recomendaciones actuales, el reemplazo de los ácidos grasos saturados por ácidos grasos mono y poliinsaturados se asocia a mejores resultados en salud. De la misma forma, este estudio apoya la eliminación de los aceites hidrogenados en los procesos industriales ya que son el principal aporte de grasas trans.

Fuente de financiamiento: beca del NIH. Conflictos de interés: El Dr. Hu declaró haber recibido financiamiento de la industria alimenticia (CaliforniaWalnut Commission and Metagenics).

\section{Comentario}

El Nurses' Health Study es una cohorte prospectiva iniciada en el año 1976 que incluye enfermeras de 30 a 55 años, por otro lado, el HPFS tiene un diseño similar, fue iniciado en el año 1986 e incluye hombres profesionales de la salud de 40 a 75 años. El presente trabajo, que recomienda disminuir el consumo de grasas saturadas y grasas trans reemplazándolos por alimentos con alto contenido de ácidos grasos mono y poliinsaturados, realizó un análisis de ambas bases de datos. Sin embargo, al analizar la calidad de la evidencia y la fuerza de su recomendación, debe considerarse que estas cohortes no poseen un grupo control, ambas tienen tiempos de seguimiento (32 años para las mujeres y 25 para los hombres) y criterios de inclusión y exclusión diferentes, y que los datos obtenidos son auto reportados. Esto podría aumentar la posibilidad de sesgo y, por lo tanto, disminuir su confiabilidad. Por otro lado, comparar los efectos en el consumo de diferentes tipos de grasas en los extremos de los quintiles (Q1 vs. Q5), sin estu'diar el consumo medio, generaría que las recomendaciones puedan no ser aplicables para la población general.
Asimismo, los reemplazos alimentarios propuestos (reemplazar grasas saturadas por grasas mono y poliinsaturadas) puede no ser factible en la práctica diaria, ya que al cambiar la manteca, crema y margarina por aceites o al reemplazar la carne vacuna por pescado de mar, las características organolépicas de las preparaciones pueden variar y no adaptarse a los gustos y preferencias de los pacientes.

\section{Conclusiones del comentador}

El presente estudio se encuentra en concordancia con las recomendaciones actuales y aporta evidencia de moderada a baja calidad para continuar con las recomendaciones vigentes sobre hábitos alimentarios.

Es importante trabajar con los pacientes para que puedan aumentar el consumo de grasas mono y poliinsaturadas a través del uso de aceites y semillas, adaptándose a sus hábitos alimentarios y, eventualmente, poder disminuir el consumo de grasas saturadas y trans.

\section{Giselle Balaciano [ Dirección de Calidad en los Servicios de Salud del Ministerio de Salud de la Nación Argentina giselle.balaciano@gmail.com]}

Balaciano G. El reemplazo de los ácidos grasos saturados por ácidos grasos mono y poliinsaturados aporta beneficios en la salud. Evid Act Pract Ambul 2016;19(4):121. Comentado de: Wang D y col. Association of Specific Dietary Fats With Total and Cause-Specific Mortality. JAMA Intern Med. 2016 Aug 1;176(8):1134-45

Referencias

1. Estudio de Salud de las Enfermeras (página institucional). Disponible en URL: http://www.nurseshealthstudy.org (último acceso 28/12/16).

2. Estudio de Seguimiento de Profesionales de la Salud (página institucional). Disponible en URL: https://www.hsph.harvard.edu/hpfs/ (último acceso 28/12/16). 